\title{
Radial tunnel syndrome in an elite power athlete: a case of direct compressive neuropathy
}

\author{
Rob D. Dickerman, Qualls E.J. Stevens, Anders J. Cohen, and S. Jaikumar \\ Surgical Neurology Branch, National Institutes of Neurological Disease and Stroke, \\ National Institutes of Health, Bethesda, Maryland
}

\begin{abstract}
Radial tunnel syndrome (RTS) is thought to result from intermittent and dynamic compression of the posterior interosseous nerve (PIN) in the proximal part of the forearm associated with repeated supination and pronation. The diagnostic criteria encompassing RTS are purely clinical and the term "radial tunnel syndrome" has become controversial because of the lack of focal motor weakness in the majority of patients diagnosed with RTS. Retrospective cadaveric and surgical studies have revealed several areas within the forearm in which the PIN may become entrapped. Recent studies have suggested that the PIN is "fixed" in the supinator muscle and that wrist pronation is the actual movement that places the most stress on the PIN. The patients most often afflicted with RTS appear to be those who perform repetitive manual tasks involving rotation of the forearm and athletes involved in racket sports. Surgical exploration with decompression of the PIN is often required in patients with RTS. We present the first case of RTS occurring in an elite power athlete and believe this case represents a direct compressive sensory neuropathy. The optimum nonsurgical treatment plan for the elite athlete in training for competition and the cause of this compressive neuropathy in power athletes will be discussed.
\end{abstract}

Key words: palsy, peripheral nerve, posterior interosseous nerve, powerlifting, supinator muscle

\section{Introduction}

In 1956, radial tunnel syndrome (RTS) was first reported as a distinct entity and described as "radial pronator syndrome" (Michele and Krueger, 1956). The term radial tunnel syndrome was coined in 1972; it defined the anatomic boundaries of the region and identified the structures that could entrap or compress the posterior interosseous nerve (PIN) (Roles and Maudsley, 1972). It was not until 20 years after recognizing RTS as a clinical syndrome that sufficient evidence was found to demonstrate compression of PIN in the radial

Address correspondence to: Rob D. Dickerman, DO, PhD, Long Island Jewish Medical Center, Department of Surgery, Division of Neurosurgery, 260-12 74th Avenue, Glen Oaks, New York 11004 USA. Tel: 516-354-3401; Fax: 516-354-8597; E-mail: drrdd@yahoo.com tunnel (Werner, 1979). RTS has been reported in swimmers, Frisbee players, tennis players, violinists, and orchestra conductors secondary to repetitive trauma related to stressful pronation and supination (Cravens and Kline, 1990). Posterior interosseous nerve entrapment is thought to occur within 4 possible structures: 1) fascial bands connecting the brachialis and brachioradialis; 2) the "leash of Henry," a fan of vessels arising from the radial recurrent artery; 3 ) the extensor carpi radialis brevis (ECRB) muscle; and 4) the arcade of Frohse, which represents the proximal border of the superficial head of the supinator muscle and is the most common cause of RTS. In contrast to previous reports of RTS occurring with repetitive stress from repeated pronosupination, we report a case of RTS occurring in a power athlete secondary to direct compression within the radial tunnel. The pathophysiology of this direct compressive RTS in an elite power athlete, the biomechanical and anatomical cause of direct compressive RTS, and 
the optimum nonsurgical treatment plan for the athlete in competitive training will be discussed herein.

\section{Case Report}

A 5'9', $255 \mathrm{lb}$. right-handed male powerlifter presented for assessment of right forearm pain. The patient described the pain as intense, sharp and debilitating during and immediately following weightlifting. He did not have loss of sensation or strength; he was training for a world powerlifting competition and stated that he was stronger than ever. The patient's training regimen was pyramidal, starting about 20 weeks before the contest and involving a weekly progression of increasing weight loads in specific lifts. The patient began having symptoms about 12 weeks into his training routine and admitted to progressive increases in discomfort over the 3 weeks before he presented at our clinic. He described the pain as intense immediately after the squat lift, which lasts for 30 to 60 seconds and slowly dissipates but remains very sensitive. He admitted that only 1 other exercise caused a similar but less severe pain, which was the hammer curl, so named for the biomechanics and resemblence of using a hammer. The movement involves bicep flexion without supination of the wrist, causing approximation of the brachioradialis and biceps. He remembered having similar pain years ago while training for a powerlifting meet, but did not seek medical attention at that time. Medical and surgical history were noncontributory. Current medications were Voltaren (diclofenac) $75 \mathrm{mg}$ bid and illicit anabolic steroids, which included methandrostenolone $30 \mathrm{mg}$ po qd, oxymetholone $50 \mathrm{mg}$ po qd, and testosterone cypionate $200 \mathrm{mg}$ IM 3 times per week. Initial examination revealed an extremely muscular male with no measurable signs of asymmetry in the upper limb musculature. There was pain and tenderness on deep palpation over the right brachioradialis and pain with resistance to extension of the extensor carpi radialis brevis (ECRB) muscle. There was no pain with supination or pronation or wrist flexion or extension. Mild pain and tenderness were elicited with deep palpation over the left brachioradialis, although there was no history of symptoms. Strength was $5 / 5$ bilaterally in all muscle groups and sensation to light touch and pinprick was intact. Deep tendon reflexes were 2/4 and symmetrical bilaterally. Spurling, Hoffman, Tinel, and Phalen tests were all negative. The patient had palpable peripheral pulses with appropriate capillary refill time, without cyanosis or clubbing of the digits and with normal skin color, texture and temperature in the upper extremities. Based on the clinical examination, a diagnosis of acute radial tunnel syndrome was made (Sarhadi et al., 1998). To further support the diagnosis and treatment options, the patient underwent a PIN block with lidocaine to the right forearm as described elsewhere (Michele and Krueger, 1956). After PIN blockade, the deep compression pain over the brachioradialis and pain with resistance to extension of the ECRB muscle was relieved. Subsequently, the patient received an injection of Decadron around the arcade of Frohse; he was urged to discontinue his illicit anabolic steroid use, avoid lifts that precipitated pain in the area, and instructed to return for reassessment in 1 week. Follow-up examination at 1 week and up to 2 months after the power meet, which he won, revealed total relief of the radial tunnel symptoms. The patient did admit that he had not been training hard since the power meet and expected the symptoms to return if he returned to the heavy powerlifting.

\section{Discussion}

Radial tunnel syndrome in power athletes has not been previously reported. The majority of reports on RTS are related to racket sports, golf, and sports involving repetitive pronosupination (Cravens and Kline, 1990). An interesting observation in this case is the occurrence of maximal pain while performing the squat. In the squat, the weight bar is resting across the upper back with maximally extended wrists, in pronation with flexed biceps (Fig. 1). This position allows for maximal compression of the brachioradialis and biceps, thus transmitting pressure into the underlying supinator and, in this case, with a load of over $1000 \mathrm{lb}$. The theory of a direct compressive neuropathy in this case is further supported by studies that have recorded pressures exerted on the nerve during stretching of the supinator muscle (Werner et al., 1980). It was demonstrated that venous blood flow decreased at pressures of 20 to $30 \mathrm{mmHg}$,

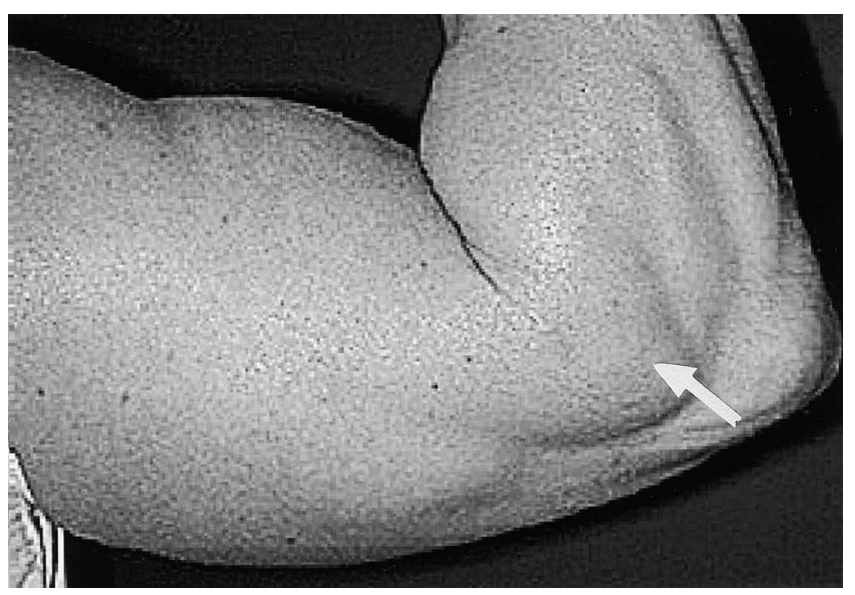

Figure 1. Positioning for direct compression of the radial nerve within the supinator muscle. Right arm with wrist maximally extended. White arrow indicates area of compression between brachioradialis and biceps. 
ischemia occurred at 60 to $80 \mathrm{mmHg}$, blockade of axonal transport at $50 \mathrm{mmHg}$, and blockade of conduction of nerve impulses occurs at 130 to $150 \mathrm{mmHg}$ (Werner et al., 1980). Our patient is able to squat over $1000 \mathrm{lb}$., which generates an enormous amount of tension and pressure in the wrist extensors, supinator and brachioradialis muscle. An earlier study on arterial pressure during weightlifting in medical students found arterial pressures of $480 / 360$ systolic/diastolic can be generated during maximal lifting (Macdougall et al., 1992). One must consider what pressures are attainable in an elite power athlete who can squat over $1000 \mathrm{lb}$. It is known that these pathophysiologic pressures lead to multiple pathological sequelae in powerlifters including pathologic concentric left ventricular hypertrophy (Dickerman et al., 1997a), pressure-overload induced hiatal hernias (Smith et al., 1999), and pathophysiologic intraocular/intracranial pressures (Dickerman et al., 1999). But can we extrapolate these pressure changes within a variety of anatomical compartments and conclude that radial nerve ischemia is occurring? It is likely, based on the fact that symptoms were worse immediately after lifting, that a reperfusion phenomenon was occurring. In addition, the arterial pressures that were documented during maximal weightlifting only lasted for 3 to 5 seconds during the valsalva maneuver (Macdougall et al., 1992). One must question whether intermittent, 3 to 5 second, supraphysiological intramuscular pressures can lead to significant nerve injury.

Earlier studies on RTS postulated that the neurological symptoms with RTS were analogous to carpal tunnel syndrome (Roles and Maudsley, 1972). A recent article has disputed such analogies and contends that the term RTS should only be used in cases of truly neurogenic cases (Rosenbaum, 1999). We agree that carpal tunnel syndrome is a poor analogy, since the majority of carpal tunnel cases involve significant motor and sensory deficits. In addition, the anatomical comparison and causes of carpal tunnel are incongruous with RTS. We believe a better anatomical and pathophysiological analogy exemplifying a direct compressive neuropathy is piriformis syndrome. Piriformis syndrome involves direct compression of the sciatic nerve as it pierces the piriformis muscle and usually manifests as a pain syndrome rather than combined sensorimotor deficits (Silver and Leadbetter, 1998).

There is no doubt anabolic steroids enhance muscle strength and can increase skeletal muscle hypertrophy (Bhasin et al., 1996). Our patient was using high doses of anabolic steroids while actively training for powerlifting competition, thus it is quite plausible that the wrist extensors and supinator muscle were undergoing hypertrophic changes induced by the weight training demands and ergogenic aids. Anabolic steroids have been reported to be directly neurotoxic when injected around peripheral nerves (Dickerman et al., 1997b). In addition, a recent report has shown that bilateral carpal tunnel developed in a bodybuilder using illicit growth hormone secondary to the soft tissue changes that occur with elevated levels of growth hormone (Dickerman et al., 2000). Our patient denied injecting anabolic steroids outside the gluteal area and denied using growth hormone. One might ask why this occurred more unilaterally in our patient. We can offer some possible answers: it occurred in his dominant hand and the patient tends to extend the wrist of the right hand more when squatting. Of course, there are internal anatomical variants within each individual that cannot be accounted for on physical exam. Of interest and in support of supinator compression are the particular exercises inducing pain; each exercise involves bicep flexion and moderate pronation, compressing the brachioradialis into the biceps and generating high pressures over the underlying supinator muscle. Thus, we postulate 2 plausible causes for RTS in this elite powerlifter: secondary to direct compression of the PIN by the hypertrophied supinator muscle; and ischemia resulting from the phenomenal pressures generated during the squats of over $1000 \mathrm{lb}$. It is likely that a combination of skeletal muscle hypertrophy and the compressive forces of the weight led to direct compression of the PIN. This report demonstrates the importance of good clinical and historical examination and how nonsurgical intervention can be an effective alternative.

\section{References}

Bhasin S, Storer TW, Berman N, Callegari C, Clevenger B, Phillips J, Bunnell TJ, Tricker R, Shirazi A, Casaburi R (1996). The effects of supraphysiologic doses of testosterone on muscle size and strength in normal men. New Engl J Med 335:1-7.

Cravens G, Kline DG (1990). Posterior interosseous nerve palsies. Neurosurgery 27:397-402.

Dickerman RD, Douglas JA, East JW (2000). Bilateral median neuropathy and growth hormone use. Arch Phys Med Rehab 81:1594-1595.

Dickerman RD, McConathy WJ, Pertussi R, Kramer W (1997a). Peripheral neuropathy and testosterone. Neurotoxicology 18:587-588.

Dickerman RD, McConathy WJ, Schaller F, Zachariah NY (1997b). Left ventricular size and function in elite bodybuilders using anabolic steroids. Clin J Sport Med 7:90-93.

Dickerman RD, Smith G, McConathy WJ, Roof LL, East JW, Smith AB (1999). Intraocular pressure changes during maximal isometric contraction: does this reflect retinal venous pressure or intracranial pressure. Neurol Res 21:243-246.

Macdougall JD, McKelvie RS, Monroz DE, Sale DG, McCartney $N$, Buick $F$ (1992). Factors affecting blood pressure during heavy weight lifting and static contractions. J Appl Physiol 73:1590-1597.

Michele AA, Krueger FJ (1956). Lateral epicondylitis of the elbow treated by fasciotomy. Surgery 39:277-284.

Roles NC, Maudsley RH (1972). Radial tunnel syndrome: resis- 
tant tennis elbow as nerve entrapment. J Bone Joint Surg Br 54B:499-508.

Rosenbaum R (1999). Disputed radial tunnel syndrome. Muscle Nerve 22:960-967.

Sarhadi NS, Korday SN, Bainbridge LC (1998). Radial tunnel syndrome: diagnosis and management. J Hand Surg 5:617-619.

Silver JK, Leadbetter WB (1998). Piriformis syndrome: assessment of current practice and literature review. Orthopedics 21: 1133-1135.
Smith AB, Dickerman RD, McGuirre S, East JE, McConathy WJ, Pearson $F$ (1999). Pressure-overload induced sliding hiatal hernias in resistance trained athletes. Clin J Gastroent 28:352-354.

Werner CO (1979). Lateral elbow pain and posterior interosseous nerve entrapment. Acta Ortho Scand Suppl 174:1-62.

Werner CO, Haeffner F, Rosen I (1980). Direct recording of local pressure in the radial tunnel during passive stretch and active contraction of the supinator muscle. Arch Orthop Trauma Surg 96:299-301. 
Copyright $\odot 2002$ EBSCO Publishing 